\title{
VO-compliant workflows and science gateways
}

\author{
G. Castellia , G. Taffoni ${ }^{\mathrm{a}}$, E. Sciacca ${ }^{\mathrm{b}}$, U. Becciani ${ }^{\mathrm{b}}$, A. Costa $^{\mathrm{b}}$, M.

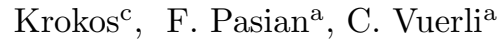 \\ ${ }^{a}$ INAF - Astronomical Observatory of Trieste, Via G.B. Tiepolo 11, 34143, Trieste, Italy \\ ${ }^{b}$ INAF - Astrophysical Observatory of Catania, Via Santa Sofia 78, 95123, Catania, Italy \\ ${ }^{c}$ School of Creative Technologies, University of Portsmouth, United Kingdom
}

\begin{abstract}
Workflow and science gateway technologies have been widely adopted by scientific communities as a valuable tool to carry out complex experiments. They offer the possibility to perform computations for data analysis and simulations, whereas hiding details of the complex infrastructures underneath. There are many workflow management systems covering a large variety of generic services coordinating execution of workflows. In this paper we describe our experiences in creating workflows oriented science gateway based on gUSE/WS-PGRADE technology and in particular we discuss the efforts devoted to develop a VOcompliant web environment.
\end{abstract}

Keywords: virtual observatory tools, scientific workflows, science gateways, computing infrastructure

\section{Introduction}

Astronomy and Astrophysics (A\&A) has become a data intensive science, due to numerous digital sky surveys across a range of wavelengths, with many terabytes of data and often with tens of measured parameters associated to each 5 observed object. Moreover new highly complex and massively large data sets are expected by novel and more complex scientific instruments and numerical simulations that will become available in the next decades and that will be

Email address: giuliano.castelli@oats.inaf.it (G. Castelli) 
largely used by the A\&A community (e.g. SKA (Taylor, 2007), CTA Acharya et al. 2013), E-ELT (Gilmozzi and Spyromilio, 2007)).

Handling and exploring these new data volumes, and actually making real scientific discoveries, poses a considerable technical challenge that requires the adoption of new approaches in using computing and storage resources and in organising scientific collaborations. To this extent workflows have emerged as a new paradigm for researchers to formalize and structure complex scientific experiments in order to enable and accelerate scientific discoveries.

Workflows system combined with Science Gateway (SGW) technologies are widely used to provide a technological framework that integrates an enriched web user interface with a solid engine to orchestrate scientific applications and tools. SGW as defined here is a community-developed set of tools, applications, and data that is integrated via a portal or a suite of applications that is further customized to meet the needs of a targeted community in a web-based graphical user interface. The computational processes supported by SGWs are organized as scientific workflows that explicitly specify dependencies among underlying tasks for orchestrating distributed resources (such as clusters, grids or clouds) appropriately.

The e-Infrastructures or Distributed Computing Infrastructures (DCI) provide a vital foundation to execute workflow's tasks and store data. In the last decade, scientific communities have adopted production DCIs to satisfy their computing and storage requirements (e.g. the European Grid Infrastructure so (EG $\left.]^{1}\right)$ and the Open Science Grid ${ }^{2}$. Managing the execution of applications on DCIs is a complex task. Moreover, solutions developed for one DCI are difficult to port to other infrastructures. In order to hide this complexity, workflow systems and SGWs are widely used as a virtualization layer on top of the underlying infrastructures (Deelman et al., 2009, Belloum et al., 2011; Barker and 35 van Hemert, 2008, Curcin and Ghanem, 2008).

\footnotetext{
${ }^{1}$ EGI: http://www.egi.eu

${ }^{2}$ Open Science Grid: www.opensciencegrid.org
} 
Research communities have developed different workflow systems and a large numbers of workflows to run their experiments (Deelman et al. 2009). These systems differ in terms of workflow description languages and workflow engines.

Workflows have been widely used also in A\&A, for example: the ESO Reflex (Freudling et al., 2013), the Astro Grid Workflow system (Winstanley, 2006), the HELIO-VO project (Bentley et al., 2011), the EU FP7 funded project Wf4Ever: Advanced Workflow Preservation Technologies for Enhanced Scienç $3^{3}$

In the framework of the ER-flow ${ }^{4}$ (Building an European Research Community through Interoperable Workflows and Data) project, the A\&A community has developed a number of workflows and SGWs for cosmological simulations, data post-processing and scientific visualisation.

While SGW and Workflows systems allow to create an environment able to benefit from DCIs, the A\&A community needs not only traditional computing resources but also the use of complex data operations that require on-line access to catalogues and archives. The Virtual Observatory provides a distributed data oriented infrastructure based on standards, tools, software and services of the International Virtual Observatory Alliance (IVOA ${ }^{5}$ ). To provide an operative environment to A\&A researchers SGWs and workflows systems should be able to interact and provide access also to $\mathrm{VO}$ tools and services.

55 In this paper we present A\&A science gateways developed using gUSE/WSPGRADE technology (Kacsuk et al. 2012) and in particular we discuss our experiences in developing VO compliant science gateways.

In the next section we present the gUSE/WS-PGRADE technology and we discuss the rationale for adopting this particular SGW framework. In section 603 we present some examples of A\&A SGW developed using the gUSE/WSPGRADE technology, then we discuss how to create a VO compliant SGW. Our results and experiences are discussed in the last section of this paper.

\footnotetext{
${ }^{3}$ Wf4Ever: http://www.wf4ever-project.org/

${ }^{4}$ ER-flow: http://www.erflow.eu

${ }^{5}$ IVOA: http://www.ivoa.net
} 


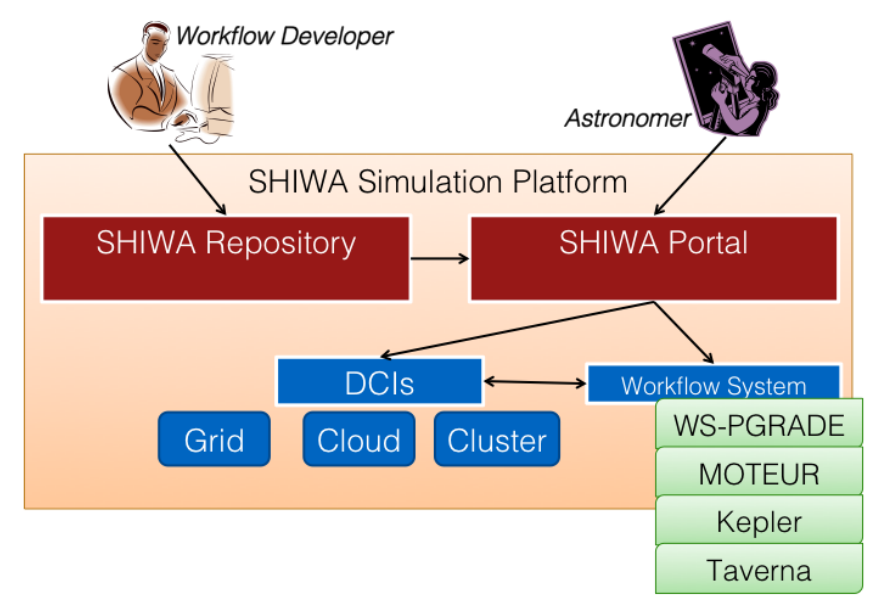

Figure 1: The SHIWA Simulation Platform. A developer designs a scientific workflow and deploys into the repository. Astronomers connect to a community portal or science gateway and import and execute the workflow. In this picture the main SHIWA simulation platform components are presented: the repository and the portal. The main components of these two services are also identified.

\section{SHIWA Workflow technology}

Workflows are a powerful mechanism to develop, execute and share scientific calculations, they can be written in graphical or text environments, and they run through a Workflow Management System (WMS): a software infrastructure to setup and execute the steps specified in the workflow description and to monitor workflows during their execution. A WMS provides the environment where in silico experiments can be defined and executed (Lin et al., 2009).

Workflows are usually designed using a modular architecture, each module being a workflow task. The dependencies between different modules are explicitly defined by the workflows visualisation and design interface (e.g. Figure 2)

In our work, we exploit the results of the SHIWA (SHaring Interoperable Workflows for large-scale scientific simulations on Available DCIs) Simulation 


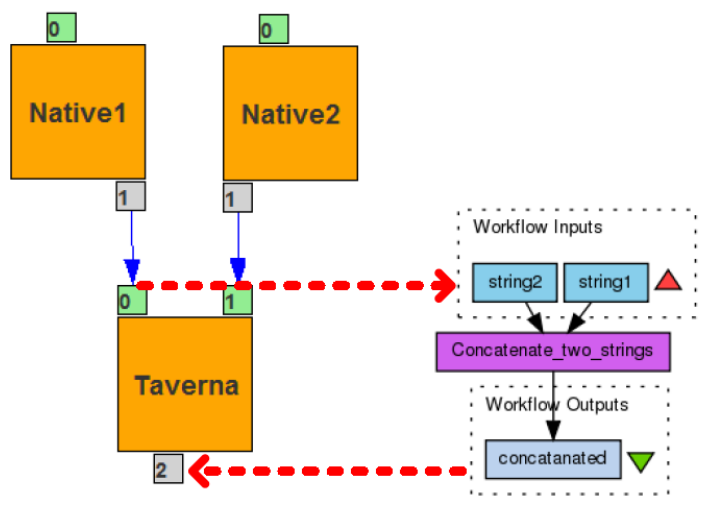

Figure 2: An example of meta-workflow. Two WS-PGRADE workflows modules (square box) are combined with a TAVERNA workflow module to concatenate two strings. Each module is actually a job to execute on the DCI. Each job communicates with other jobs within the workflow through job-owned input and output ports. An output port (small grey boxes) of a job connected with an input port (small green boxes) of a different job is called channel (blue arrow); these are directed edges of the graph. A single port must be either an input, or an output port of a given job.

75 Platform (SSP) ${ }^{6}$ (Terstyanszky et al., 2014) to implement sharing and exchanging of workflows between workflow systems and DCI resources through the SSP.

gUSE/WS-PGRADE framework is a widely adopted robust technology. A number of scientific communities are using this framework to build their SGW (e.g. CancerGrid gateway, ProSim gateway, Amsterdam Medical Centre gateway and the MosGrid gateway) as discussed by Kacsuk et al. (2012).

The main reason we adopt this technology it that SSP allows workflow developers to design a workflow that combines together modules written for different WMS as shown in Figure 2 The SSP adopted the Coarse-grained interoperability concept (Kukla et al. 2008): different workflow systems are nested to achieve interoperability of execution frameworks. In the SSP the non-native workflows are used as "black boxes" to be embedded into a so called meta-worflow that invokes external workflow engines.

\footnotetext{
6 http://www.shiwa-workflow.eu
} 
From the user's perspective the most important services offered by the SSP are shown in Figure 1 .

- The SHIWA Repository 7 A database where workflows and meta-data about workflows can be stored. The database is a central repository for users to discover and share workflows within and across their communities.

- The SHIWA Porta 8 A web portal integrated with the SHIWA Repository that enables the execution of SHIWA repository workflows.

The SHIWA Portal is based on the gUSE/WS-PGRADE technology. gUSE/WSPGRADE is a collaborative and community oriented application development environment that allows developers and end-users to develop and share workflows, workflow graphs, workflow templates, and ready-to-run workflow applications. It is based on Liferay 9 , a portal framework which is highly customizable due to the adoption of JAVA portlet technology. The main software components of gUSE/WS-PGRADE are:

- gUSE is a resource virtualization environment that allows execution of workflow modules on a variety of DCIs (e.g. desktop grids, clouds, clusters and web service).

- WS-PGRADE is a workflow engine (Kacsuk, 2011) that offers generic services to handle distribution, monitoring and execution of workflows modules.

WS-PGRADE is the Master Workflow System of the SHIWA Portal, workflows developed using WS-PGRADE are called native workflows. However, SSP is not limited in executing only native workflows, but thanks to the "Grid Execution Management for Legacy Code Applications" (GEMLCA) job wrapper (Delaitre et al., 2005), it is able to run workflows written using differ workflow

${ }^{7}$ http://shiwa-repo.cpc.wmin.ac.uk

8 http://shiwa-portal2.cpc.wmin.ac.uk/liferay-portal-6.1.0

http://www.liferay.com 
languages requiring different WMSs (non-native workflows). A meta-workflow is a workflow that involves both native and non-native workflows as its constituent parts. The ability to design and execute meta-workflows is a peculiar characteristic of SSP and we will use it to develop our SGWs and portal.

Through the SHIWA Portal a scientist can define and run simulations on various DCIs, including the European Grid Infrastructure, but also local clusters and Cloud infrastructures (see Figure 1). The portal (via third party workflow engines) provides support for a number of commonly used academic workflow engines (e.g MOTEUR (Glatard et al., 2008), Taverna, Kepler) and it can be extended with other engines.

In Figure 2, we present an example of meta-workflow where a Taverna based module is combined with WS-PGRADE native workflows.

The generic WS-PGRADE portal instance is easily customizable into a research domain specific science gateway thanks to a particular portal extension called Application Specific Module (ASM) (Balasko et al. 2010). ASM consists of two components: a) a script-layer used for installing different parts of the module (e.g. data tables, services, portlets) and b) the Java-layer used as the Application Programming Interface (API) during the development of the web-interface providing programmatically most of the functionalities of gUSE.

Using JAVA portlets it is possible to develop a web user interface to provide input parameters, to execute applications and to display the results in a userfriendly way. Each application specific portlet contains the details of the related underlying workflows.

Moreover, using the SSP it is possible to design and implement a SGW able to execute meta-workflows that combine together native and non-native workflow modules.

\section{A\&A Science Gateways and workflows}

In the last decade, scientific workflows are playing an important role in the working methodology of the A\&A community. The SSP is not the first and only 


\section{Standard Gravity Model}

Modified Gravity Model

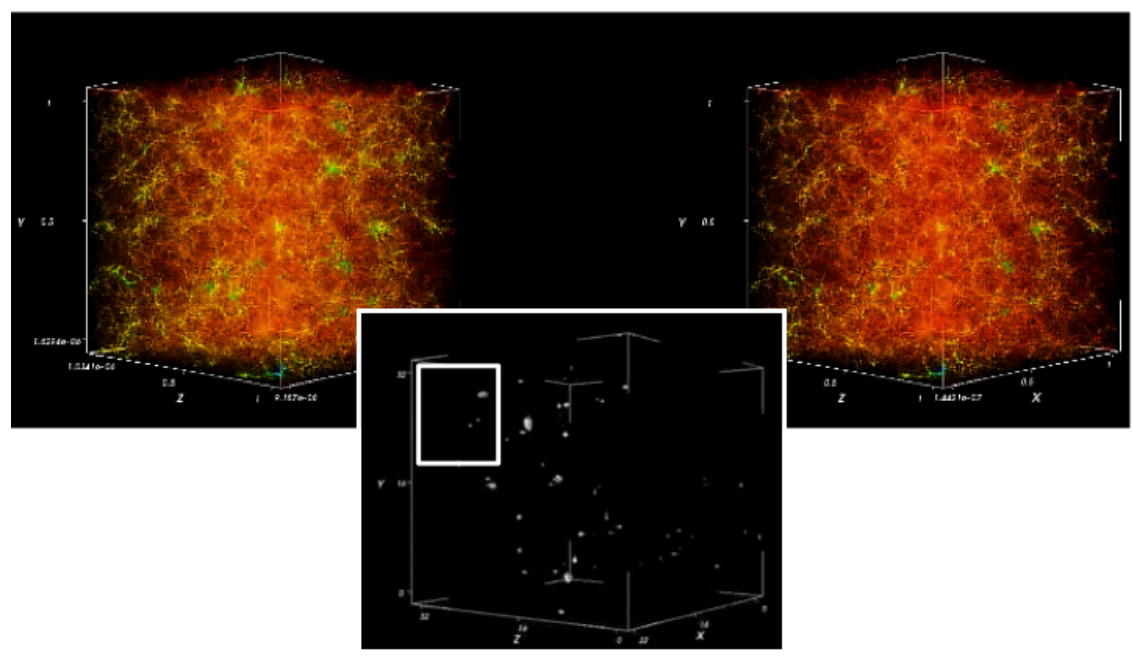

Figure 3: Studying the acceleration of the Universe with the LaSMoG workflow: visual comparison of a standard gravity model and a modified gravity model (i.e. without introducing dark energy) taking advantage of the VisIVO visualisation. Using VisIVO it is possible to plot 3D box from two N-Body simulations at the same redshift that starts from the same initial conditions. One simulation is a $\Lambda$ CDM with standard gravity (left) the other is a modified gravity model (right). In the modified gravity simulation the Cosmic structures collapse at higher redshift. 
workflow technology used by astronomers, as an example here we can cite the ESO Reflex, Pegasus and the AstroGrid workflows system (see Schaaff, A. and Ruiz J.E. (2013) for a more complete discussion on Workflows in A\&A).

The European Southern Observatory ( $\mathrm{ESO}^{10}$ Recipe flexible execution workbench (Reflex) (Freudling et al., 2013), an environment to automate data reduction workflows. Reflex is implemented as a package of customized components for the Kepler (Ludascher et al. 2006) workflow engine. Kepler provides the graphical user interface to create an executable flowchart-like representation of the data reduction process. Key features of Reflex are: a rule-based data organiser, an infrastructure to re-use results, interactive user interfaces, and a novel concept to exploit information created during data organisation for the workflow execution.

Pegasus is a WMS (Deelman et al. 2014) that allows scientists to construct workflows in abstract terms without worrying about the details of the underlying execution environment. It allows the execution of workflows on different computing infrastructures (from local cluster up to DCIs), and it is widely used both in the framework of the OSG and of the XSEDE HPC infrastructure.

The AstroGrid Workflow System was developed in the framework of the UK Virtual Observatory System. It was a multi-user batch system for the execution of potentially long-running astronomical workflows based on a description file that lists the remote applications and data to use. It was based on the Common Execution Architecture Web service interface, message protocols, and formats and it used the VO standards to execute tasks on VO compliant applications and resources.

These systems differ in terms of workflow description languages and workflow engines. This often has a profound impact on the resulting workflow performance, development effort, management and portability. It takes significant effort and time to learn how to use workflow systems, and requires specific expertise and skills to develop and maintain workflows. Moreover, workflows

${ }^{10}$ ESO: http://www .eso.org 
developed in one system cannot be executed using a different one.

As Pegasus WMS, the SSP allows to execute workflows tasks on different resources and to develop SGWs, but it also provides a workflow repository able to store workflows form different WMS and that can be used as a mean of workflows preservation. More importantly, differently from any other platform, the SSP allows workflows interoperability giving the possibility to re-use workflows written using differ WMS.

Using the SSP and the gUSE/WS-PGRADE technology we developed several SGWs and associated workflows focused on different A\&A applications (Becciani et al. 2014a) as below:

1. COMCAPT (Capture of comets from the interstellar space by the Galactic tide), provided by the Astronomical Institute of Slovak Academy of Sciences. It is a SGW that focuses on applications related to studies of small bodies in the Solar system.

2. FRANEC (Frascati Raphson Newton Evolutionary Code), provided by INAF - Osservatorio Astronomico di Teramo. It allows to execute stellar evolutionary code on a DCI (Taffoni et al. 2010).

3. LaSMoG (Large Simulation for Modified Gravity), provided by the University of Portsmouth (UK) (Zhao et al., 2011). It supports the LaSMoG consortium to investigate large-scale modified gravity models, more specifically inspecting datasets to discover anomalies by comparing suitably with datasets coming from standard models (i.e. dark energy models).

4. MESTREAM (Modelling the dynamical Evolution of meteoroid stream), provided by Astronomical Institute of Slovak Academy of Sciences. This SGW allows Astronomers to calculate the dynamical Evolution of meteoroid streams.

5. Planck (Simulations of the ESA Planck satellite mission), provided by INAF - Osservatorio Astronomico di Trieste. This SGW is designed to execute simulation of Planck satellite mission developing a web application of the Planck simulation software (Reinecke et al. 2006). 
6. VisIVO (Visualisation Interface for the Virtual Observatory) (Sciacca et al., 2013), provided by INAF - Osservatorio Astrofisico di Catania (Figure 4). It provides visualisation and data management services to the scientific community exploiting the functionalities of VisIVO (Becciani et al., 2010). that do not require strong computing resources.

Finally each SGW is equipped with a Data Management portlet (see Figure

\footnotetext{
${ }^{11}$ EMI grid infrastructures: http://www.eu-emi.eu/
} 


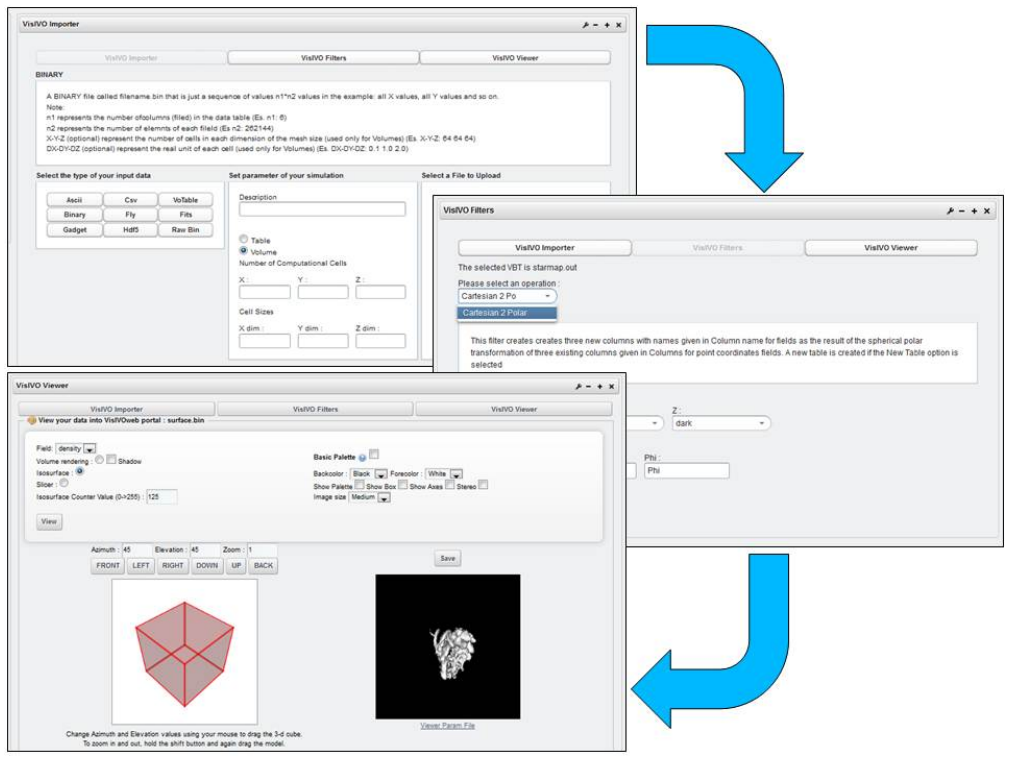

Figure 4: VisIVO Science Gateway provides visualisation and data management services to the scientific community thanks to the VisIVO visualisation framework.

5) that allows users to access their private staging area where input and output files are produced. The Data Management portlet interfaces not only with the docal server filesystem but also with EMI LFC distributed filesystem (Laure et al. 2006).

\section{VO oriented workflows}

While the Astro SGWs described before are strictly computing oriented, Astronomers need also to access data using IVOA standards and services. Commonly workflows orchestrate computing and storage tasks running on a computing infrastructure (a local server, a cluster or a DCI). However, also the VO is a DCI: it is a data and services infrastructure. For this reason, we adopt VO workflow components, that allow to create workflows implementing a high level

of modularity combining together different DCIs. 


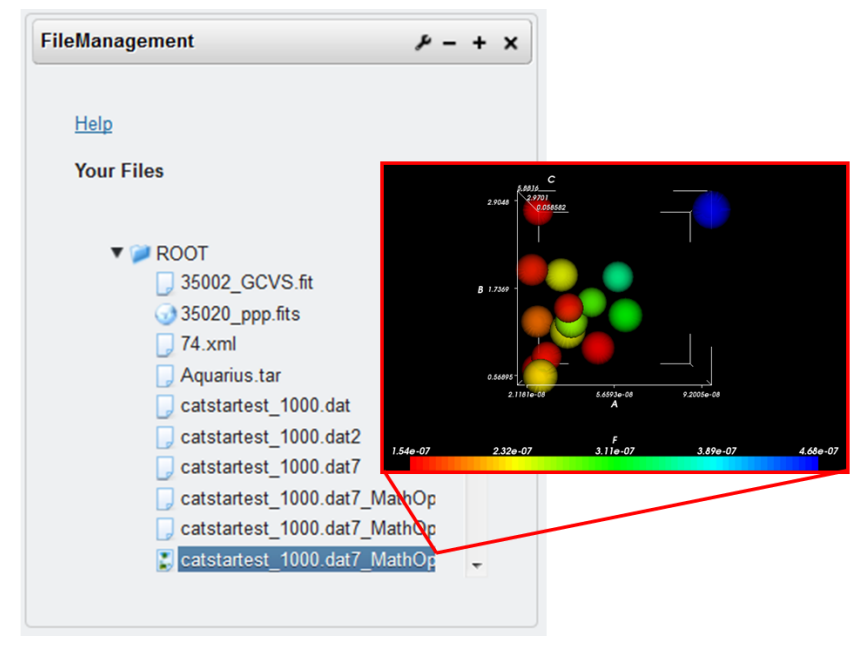

Figure 5: Data Management portlet: it allows to access users private staging area, input and output files.

In the framework of Workflow4Ever project ${ }^{12}$ the Astrophysics community has developed more than 50 workflows using Taverna $2^{13}$ (Wolstencroft et al. 2013 ) and the AstroTaverna ${ }^{14}$ plugin (Schaaff et al. 2012). AstroTaverna integrates existing Virtual Observatory web services as first-class building blocks in Taverna workflows (e.g. to search a registry, to add identified services to the workflow, to manipulate data in form of VOTable: 15 and to convert coordinates). These workflows are used to interact with data, focusing on searching and getting data in distributed database systems, manipulating data or performing simple data analysis tasks.

To develop a SGW that allows also to implement data access we use SSP capability of executing non-native workflows.

AstroTaverna provides the means to build A\&A workflows using Virtual Observatory discovery services including manipulation of VOTables (based on

\footnotetext{
${ }^{12}$ Workflow4Ever project: http://www.wf4ever-project.org

${ }^{13}$ Taverna: http://www.taverna.org.uk

${ }^{14}$ AstroTaverna: http://amiga.iaa.es/p/290-astrotaverna.htm

${ }^{15}$ VOTable standard: http://www.ivoa.net/documents/VOTable/
} 
STIL tool set). It integrates SAMP-enabled software (Taylor et al., 2012),

\footnotetext{
${ }^{16}$ MyExperiment Taverna workflow repository: http://www .myexperiment.org

${ }^{17} \mathrm{~A}$ Cone is a circular region on the sky defined by a sky position and a radius around that position. A Cone Search is a query for information related to a Cone

${ }^{18}$ SimDM: http://www.ivoa.net/documents/SimDM/
} 
use of Workflows in the VO (Schaaff, A. and Ruiz J.E. 2013). They identify different ways in which workflows can be used that implies different levels of involvement of the VO services, from the simple case when a pipeline requests VO-compliant data, up to the complex case when the VO is used to drive the 285 job remotely and manage the results.

To this extent, different VO standards or recommendations are involved. In fact to drive jobs remotely from the VO it is al lest necessary to rely on a SingleSign-On system (SSO) (Rixon G. and Graham M., 2008) and to the Universal Worker Service (UWS) (Harrison P. and Rixon G., 2014). The SSO is necessary to authenticate and authorise the users on the remote resources while the UWS pattern defines how to manage asynchronous execution of jobs on the remote systems.

Moreover, to manage the results of the workflows execution it is necessary to access a distributed storage. In the VO framework the interface over the distributed storage is the VOSpace (Graham et al., 2014).

On the other hand, in our work we focus on the interoperability aspects between computing and VO DCIs. Our workflows use the computing and storage resource offered by computing infrastructures and the VO services as regards data access and manipulation. The SGWs developed using SSP can be configured to access a variety of DCIs thanks to the gUSE component. Actually it is missing a gUSE UWS plug-in, however at this stage the DCIs we are using does not require it. The SGWs allow to implement different SSO systems, we choose the one that are compliant with the VO SSO specifications in our case we are using TLS with passwords and openID. The SSP allows to implement also other SSO mechanisms some of them as SAML/Shibboleth are under discussion by the Grid and Web Service working group.

The SSP provides a methods for credential delegation used to delegate a user's credentials to remote resources for example to allow data transfer on behalf of the user. This SSP capability could be integrated to be compliant 310 with the IVOA credential delegation protocol.

A key aspect to increase the interoperability between with the VO is the 
possibility to manage the results of the workflows execution also to the VO. The SSP is not providing a VOspace interface or client that allows to store data on VOspace compliant distributed storage. A gUSE plug-in will be developed to overcome this limitation.

\section{Discussion and Conclusions}

In the last four years, a large effort has been devoted to develop SGWs and workflows for A\&A applications. Our efforts allow to set up a number of SGWs that provide the A\&A community with a set of tools towards facilitating the use of DCIs by demonstrating benefits of using this approach in doing science.

Developing a SGW poses some important challenges, regarding DCI access, adoption of a suitable web technology, user authorisation and authentication and so on. In practice, the major problem in the development of a SGW can be summarised as how to rapidly deploy scientific applications on computational resources and expose these applications as web services to scientists.

In this paper, we adopt a web framework that simplifies the development of SGW: the SSP. This framework allows developers to host their domain-specific software application and rapidly generate SGW interfaces to them. The application must be structured in terms of workflows. Workflows encapsulate a formal specification of a scientific process and they highlight and automate the analytical and computational steps of any scientific application.

According to our experiences, SSP provides a stable and secure framework for developers that takes care of all the problems related to the interfacing with DCIs, including the web development and the applications porting. It is widely used by various scientific communities (Kacsuk et al. 2012) and it allows to deploy SGWs that implement meta-workflows.

This framework allows to select the computing and storage back-end of the SGWs according to the computing and storage needs of the single tasks of the application or workflow module. We tested in particular local computing 
performances.

Thanks to SSP, workflows and portlets have been built and integrated into the SGWs and they have been successfully made available to users. The new SGWs enable researchers to facilitate the usage of large scale computing system as DCI for exploring a wide range of parameter values and comparing the outcomes.

From our experience in building SGWs, we believe that SSP captures a common pattern in the software architecture of SGWs and can be applied to a variety of different applications.

Bearing in mind that the main aim of the International VO Alliance is to make both observational and theoretical data more easily accessible to the whole scientific community an important aspect regards the integration of the VO data infrastructure within the A\&A SGWs. Thanks to the meta-workflow capability of SSP, we are able to implement AstroTaverna modules into our gateways giving the possibility to create a SGW able to access and produce data using VO tools and services.

A workflow written under different WMS can be recycled allowing us to deploy AstroTaverna workflows thus optimising our efforts in achieving VOcompliant SGWs. Although the major obstacle of workflow re-cycling is that workflow systems are not normally compatible, our adoption of SSP framework allows to overcome this limitation. We notice that this approach improves efficiency and reliability by reusing tested methodologies, it increases the lifetime of workflows and it reduces development time for new workflows and consequently SGWs. Interoperability among workflow systems does not only permit the development and enhancement of large-scale and comprehensive workflows, but also reduces the existing gap between different DCIs, and consequently promotes cooperation among research communities exploiting these DCIs.

In the framework of the ER-flow project 19 , we have received a very positive feedback from the SGW users showing that this approach is a promising towards

${ }^{19}$ ER-flow project: http://www.erflow.eu 

improve SGWs functionalities. The planned future developments for the A\&A SGWs in the framework of the Italian Virtual Observatory initiative VObs.it, are the following:

- Cloud infrastructures. We wish to include new DCIs as SGW back-end, in

${ }^{20}$ EGI Federated Cloud: http://go.egi.eu/cloud 
and it involves a number of European institutions.

\section{Acknowledgement}

The research leading to these results has received funding from the FP7

400

Community through Interoperable Workflows and Data) and the European Commission's Seventh Framework Programme (FP7/2007-2013) under grant agreement no 283481 SCI-BUS (SCIentific gateway Based User Support).

\section{References}

Acharya, B., Actis, M., Aghajani, T., Agnetta, G., et al., 2013. Introducing the CTA concept. Astroparticle Physics 43, 3 - 18. Seeing the High-Energy Universe with the Cherenkov Telescope Array - The Science Explored with the CTA.

Balasko, A., Kozlovszky, M., Schnautigel, A., Karóckai, K., Márton, I., Strodl, 410 T., Kacsuk, P., 2010. Converting P-GRADE grid portal into e-science gateways. International Workshop on Science Gateways , 1-6.

Barker, A., van Hemert, J., 2008. Scientific workflow: A survey and research directions, in: Wyrzykowski, R., Dongarra, J., Karczewski, K., Wasniewski, J. (Eds.), Parallel Processing and Applied Mathematics. Springer Berlin Heidelberg. volume 4967 of Lecture Notes in Computer Science, pp. 746-753.

Becciani, U., Costa, A., Antonuccio-Delogu, V., Caniglia, G., Comparato, M., Gheller, C., Jin, Z., Krokos, M., Massimino, P., 2010. Visivo-integrated tools and services for large-scale astrophysical visualization. Publications of the Astronomical Society of the Pacific 122, 119-130.

420

Becciani, U., Sciacca, E., Costa, A., Massimino, P., Pistagna, C., Riggi, S., Vitello, F., Petta, C., Bandieramonte, M., Krokos, M., 2014a. Science gateway technologies for the astrophysics community. Concurrency and Computation: Practice and Experience . 
Becciani, U., et al., 2014b. Creating gateway alliances using ws-pgrade/guse, in: Science Gateways for Distributed Computing Infrastructures. Springer, pp. $255-270$.

Belloum, A., Inda, M., Vasunin, D., Korkhov, V., Zhao, Z., Rauwerda, H., Breit, T., Bubak, M., Hertzberger, L., 2011. Collaborative e-science experiments and scientific workflows. Internet Computing, IEEE 15, 39-47.

Bentley, R., Brooke, J., Csillaghy, A., Fellows, D., Le Blanc, A., Messerotti, M., Perez-Suarez, D., Pierantoni, G., Soldati, M., 2011. Helio: Discovery and analysis of data in heliophysics, in: E-Science (e-Science), 2011 IEEE 7th International Conference on, pp. 248-255. doi 10.1109/eScience.2011.42.

Bonnarel, F., Fernique, P., Bienaymé, O., Egret, D., Genova, F., Louys, M., Ochsenbein, F., Wenger, M., Bartlett, J.G., 2000. The ALADIN interactive sky atlas. A reference tool for identification of astronomical sources. A\&A $143,33-40$.

Curcin, V., Ghanem, M., 2008. Scientific workflow systems - can one size fit all?, in: Biomedical Engineering Conference, 2008. CIBEC 2008. Cairo International, pp. 1-9.

Deelman, E., Gannon, D., Shields, M., Taylor, I., 2009. Workflows and e-science: An overview of workflow system features and capabilities. Future Generation Computer Systems 25, $528-540$.

Deelman, E., Vahi, K., Juve, G., Rynge, M., Callaghan, S., Maechling, P.J., Mayani, R., Chen, W., da Silva, R.F., Livny, M., Wenger, K., 2014. Pegasus, a workflow management system for science automation. Future Genq eration Computer Systems, -doi/http://dx.doi.org/10.1016/j.future. 2014.10 .008

Delaitre, T., Kiss, T., Goyeneche, A., Terstyanszky, G., Winter, S., Kacsuk, P., 450 2005. Gemlca: Running legacy code applications as grid services. Journal of Grid Computing 3, 75-90. 
Freudling, W., Romaniello, M., Bramich, D.M., Ballester, P., Forchi, V., García-

Dabló, C.E., Moehler, S., Neeser, M.J., 2013. Automated data reduction workflows for astronomy. The ESO Reflex environment. A\&A 559, A96.

Gilmozzi, R., Spyromilio, J., 2007. The European Extremely Large Telescope (E-ELT). The Messenger 127, 11.

Glatard, T., Montagnat, J., Lingrand, D., Pennec, X. (Grid'5000), 2008. Flexible and efficient workflow deployement of data-intensive applications on grids with MOTEUR. International Journal of High Performance Computing Applications 22, 347-360. Special issue on Workflow Systems in Grid Environments.

Graham, M., Morris, D., Rixon, G., Dowler, P., Schaaff, A., Tody, D., Ma-

口 jor, B., 2014. Vospace service specification. URL: http://www.ivoa.net/ documents/VOSpace/.

465 Harrison P. and Rixon G., 2014. Universal worker service pattern. URL: http: //wWW.ivoa.net/documents/UWS/index.html

Kacsuk, P., 2011. P-GRADE portal family for grid infrastructures. Concurrency and Computation: Practice and Experience 23, 235-245.

Kacsuk, P., Farkas, Z., Kozlovszky, M., Hermann, G., Balasko, A., Karoczkai, K., Marton, I., 2012. WS-PGRADE/gUSE Generic DCI gateway framework for a large variety of user communities. Journal of Grid Computing 10, 601630 .

Kukla, T., Kiss, T., Terstyanszky, G., Kacsuk, P., 2008. A general and scalable solution for heterogeneous workflow invocation and nesting, in: Workflows in Support of Large-Scale Science, 2008. WORKS 2008. Third Workshop on, pp. $1-8$.

Laure, E., Gr, C., Fisher, S., Frohner, A., Kunszt, P., Krenek, A., Mulmo, O., Pacini, F., Prelz, F., White, J., Barroso, M., Buncic, P., Byrom, R., Cornwall, 
L., Craig, M., Meglio, A.D., Djaoui, A., Giacomini, F., Hahkala, J., Hemmer, F., Hicks, S., Edlund, A., Maraschini, A., Middleton, R., Sgaravatto, M., Steenbakkers, M., Walk, J., Wilson, A., 2006. Programming the grid with glite, in: Computational Methods in Science and Technology, p. 2006.

Lin, C., Lu, S., Fei, X., Chebotko, A., Pai, D., Lai, Z., Fotouhi, F., Hua, J., 2009. A reference architecture for scientific workflow management systems and the view soa solution. IEEE Transactions on Services Computing 2, 79-92.

Ludascher, B., Altintas, I., Berkley, C., Higgins, D., Jaeger, E., Jones, M., Lee, E.A., Tao, J., Zhao, Y., 2006. Scientific workflow management and the kepler system. special issue: workflow in grid systems. Concurr. Comput.: Pract. Exp , 1039-1065.

Reinecke, M., Dolag, K., Hell, R., Bartelmann, M., Enßlin, T.A., 2006. A simulation pipeline for the Planck mission. A\&A 445, 373-373.

Rixon G. and Graham M., 2008. Ivoa single-sign-on profile: Authen-

q tication mechanisms. URL: http://www.ivoa.net/documents/latest/ SSOAuthMech.html

Schaaff, A., et al., 2012. Scientific Workflows in Astronomy, in: Astronomical Data Analysis Software and Systems XXI, p. 875.

Schaaff, A. and Ruiz J.E., 2013. Scientific workflows in the vo. URL: http: //www.ivoa.net/documents/Notes/ScientificWorkflows/index.html.

Sciacca, E., Bandieramonte, M., Becciani, U., Costa, A., Krokos, M., Massimino, P., Petta, C., Pistagna, C., Riggi, S., Vitello, F., 2013. Visivo science gateway: a collaborative environment for the astrophysics community, in: 5th International Workshop on Science Gateways, IWSG 2013, CEUR Workshop Proceedings. pp. $1-8$.

Taffoni, G., Cassisi, S., Manzato, P., Molinaro, M., Pasian, F., Pietrinferni, 505 A., Salaris, M., Vuerli, C., 2010. Grid and databases: Basti as a practical integration example. Journal of Grid Computing 8, 223-240. 
Taylor, A.R., 2007. The square kilometre array, in: A Giant Step: from Millito Micro-arcsecond Astrometry, pp. 164-169.

Taylor, M.B., Boch, T., Fay, J., Fitzpatrick, M., Paioro, L., 2012. SAMP: Application Messaging for Desktop and Web Applications, in: Ballester, P., Egret, D., Lorente, N.P.F. (Eds.), Astronomical Data Analysis Software and Systems XXI, p. 279.

Terstyanszky, G., Kukla, T., Kiss, T., Kacsuk, P., Balasko, A., Farkas, Z., 2014. Enabling scientific workflow sharing through coarse-grained interoperability. Future Generation Computer Systems 37, 46 - 59. Special Section: Innovative Methods and Algorithms for Advanced Data-Intensive Computing Special Section: Semantics, Intelligent processing and services for big data Special Section: Advances in Data-Intensive Modelling and Simulation Special Section: Hybrid Intelligence for Growing Internet and its Applications.

${ }_{520}$ Winstanley, N., 2006. Design and implementation of the astroa grid workflow system. URL: http://www.ivoa.net/documents/latest/ AstrogridWorkflow.html.

Wolstencroft, K., Haines, R., Fellows, D., Williams, A.R., Withers, D., Owen, S., Soiland-Reyes, S., Dunlop, I., Nenadic, A., Fisher, P., Bhagat, J., Belhajjame, ${ }_{525}$ K., Bacall, F., Hardisty, A., de la Hidalga, A.N., Vargas, M.P.B., Sufi, S., Goble, C.A., 2013. The taverna workflow suite: designing and executing workflows of web services on the desktop, web or in the cloud. Nucleic Acids Research , 557-561.

Zhao, G.B., Li, B., Koyama, K., 2011. N-body simulations for f (r) gravity using a self-adaptive particle-mesh code. Physical Review D 83, 044007. 